

\section{REVISTA \\ TEORÍA Y PRÁCTICA \\ DE LA \\ ARQUEOLOGÍA HISTÓRICA LATINOAMERICANA}

ISSN: 2250-866X (impreso) | ISSN: 2591-2801 (en línea)

AÑO VIII, VOLUMEN 9, PRIMAVERA DE 2019

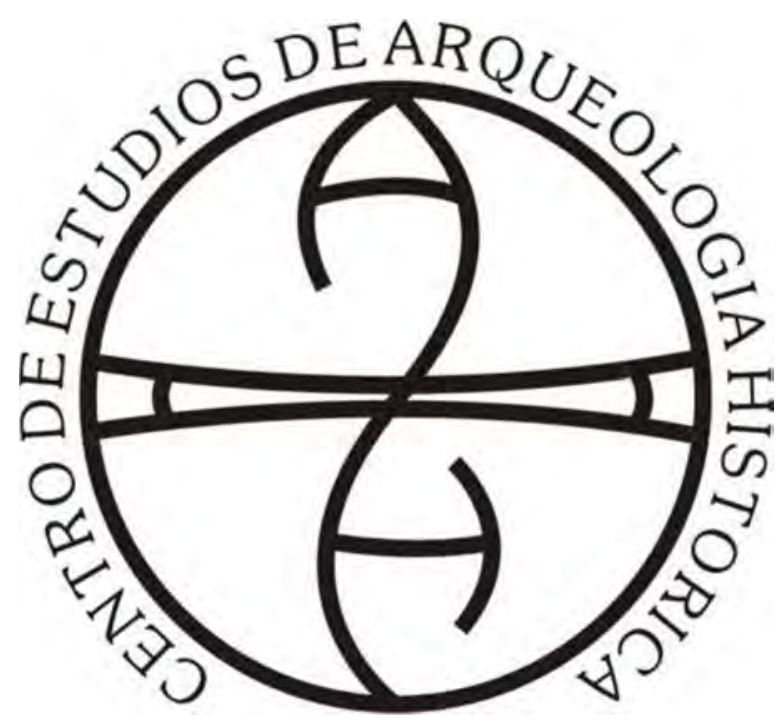

CENTRO de Estudios de ARqueOlOGía HistóricA

FACULTAD DE HUMANIDADES Y ARTES | UNIVERSIDAD NACIONAL DE ROSARIO 
(Universidad Nacional de Rosario, Universidad Nacional de Río Cuarto,

Universidad Nacional de San Juan, Universidad de la República, Universidad Nacional de Trujillo)

\author{
AUTORIDADES DE LA UNIVERSIDAD NACIONAL DE ROSARIO \\ RECTOR: Lic. Franco Bartolacci \\ VICE-RECTOR: Od. Darío Macía \\ SECRETARIO GENERAL: Prof. José Goity \\ SECRETARIA ACADÉMICO Y DE APRENDIZAJE: Dr. Marcelo Vedrovnik \\ SECRETARÍA DE CIENCIA TECNOLOGÍA E INNOVACIÓN \\ PARA EL DESARROLLO: Ing. Guillermo Montero.
}

\author{
AUTORIDADES DE LA FACULTAD DE HUMANIDADES Y ARTES \\ DECANO: Prof. Alejandro Vila \\ VICEDECANA: Prof. Marta Varela \\ SECRETARIA ACADÉMICA: Dra. Marcela Coria
}

\author{
AUTORIDADES DEL CENTRO DE ARQUEOLOGÍA HISTÓRICA \\ DIRECTORA: Dra. Ana Rocchietti \\ SECRETARIA: Prof. Nélida de Grandis \\ PROSECRETARIA: Lic. Marianela Bizcaldi
}

DIRECTORAS - EDITORAS:

Dra. Ana Rocchietti y Prof. Nélida De Grandis

SECRETARIA DE EDICIÓN: Dra. Irene Dosztal

Este número es co-edición de las ponencias

del VIII Congreso Nacional de Arqueología Histórica (2018) entre:

Centro de Estudios en Arqueología Histórica: Directora Ana Rocchietti

Centro de Estudios en Arqueología Regional: Director Fernando Oliva

Centro de Estudios en Arqueología Subacuática: Directora Mónica Valentini

Departamento de Arqueología, Escuela de Antropología: Director Fernando Oliva
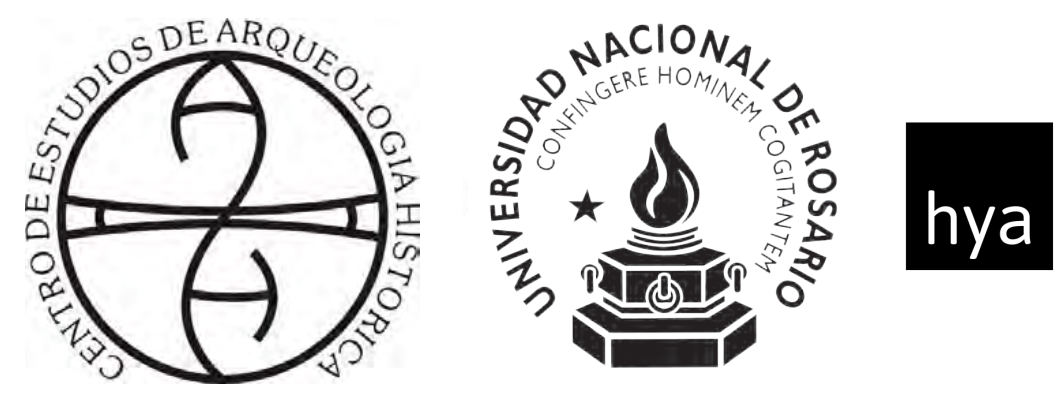

Facultad de Humanidades

y Artes_UNR
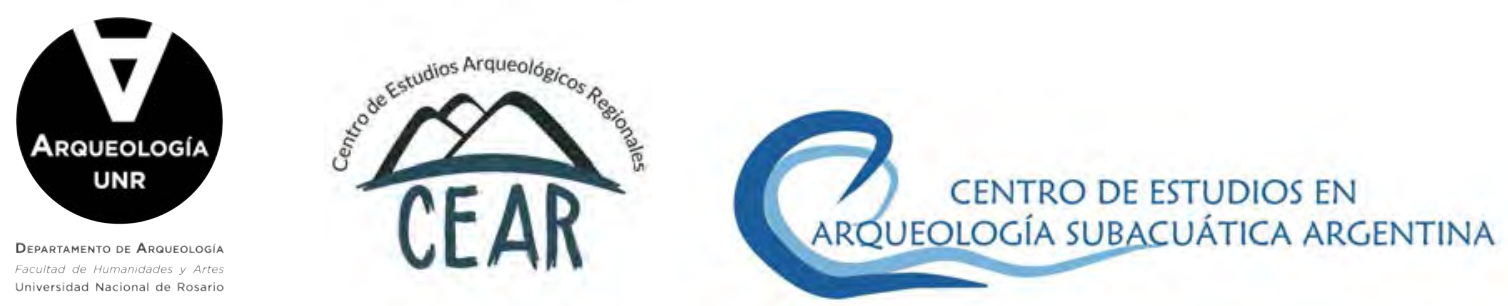
Comité Permanente de los Congresos Nacionales de Arqueología Histórica

Dr. Daniel Schávelzon (Universidad Nacional de

Buenos Aires)

Prof. María Teresa Carrara (Universidad Nacional de Rosario)

Prof. Carlos Baldassarre (Museo Municipal de Río

Grande, Tierra del Fuego) in memoriam

Dr. Mariano Ramos (Universidad Nacional de Luján, CONICET)

Dr. Horacio Chiavazza (Universidad Nacional de Cuyo)

Dra. Ana María Rocchietti (Universidad Nacional de

Rosario, Universidad Nacional de Río Cuarto)

Lic. Facundo Gómez Romero (Universidad Autónoma

de Barcelona)

\section{Comité Científico}

Dra. Tânia Andrade Lima (Universidade Federal do Rio de Janeiro)

Prof. Réginald Auger (CELAT/Département des

Sciences Historiques, Université Laval, Canadá)

Dr. Roberto Bárcena (Universidad Nacional de Cuyo, CONICET)

Dra. Marta Bonaudo (Universidad Nacional de Rosario, CONICET)

Dr. Leonel Cabrera (Universidad de la República, Uruguay)

Dr. Luis María Calvo (Universidad Católica de Santa

$\mathrm{Fe})$

Prof. Juan Castañeda Murga (Universidad Nacional de

Trujillo, Perú)

Dr. Carlos Ceruti (Museo de Ciencias Naturales y

Antropología "Prof. Antonio Serrano”. Paraná)

Dr. Horacio Chiavazza (Universidad Nacional de Cuyo)

Dra. Silvia Cornero (Universidad Nacional de Rosario)

Prof. Pedro Paulo Funari (Universidade Estadual de

Campinas, Brasil)

Lic. Jorge A. Gamboa Velásquez (Universidad Nacional

Santiago Antuñez de Mayolo, Perú)

Dr. Eduardo Alejandro García (Universidad Nacional de

San Juan, CONICET)

Prof. Nélida De Grandis (Universidad Nacional de

Rosario)

Dr. Juan Bautista Leoni (Universidad Nacional de

Rosario, CONICET)

Dra. Amancay Martínez (Universidad Nacional de San

Luis)

Dra. Catalina Teresa Michieli (Universidad Nacional de

San Juan, CONICET)

Lic. Fernando Oliva (Universidad Nacional de Rosario)
Ing. Adrián Pifferetti (Universidad Tecnológica Nacional Regional Rosario)

Dr. Mariano Ramos (Universidad Nacional de Luján, CONICET)

Dra. Ana María Rocchietti (Universidad Nacional de Rosario)

Dr. Daniel Schávelzon, (Universidad Nacional de Buenos Aires, CONICET)

Dra. Carlota Sempé (Universidad Nacional de La Plata)

Dr. Mario Silveira (Universidad Nacional de Buenos

Aires)

Dra. Silvia Simonassi (Universidad Nacional de Rosario)

Dra. Alicia Tapia (Universidad Nacional de Buenos Aires, Universidad Nacional de Luján)

Lic. Mónica P. Valentini (Universidad Nacional de Rosario)

Agrim. Benito Vicioso (Universidad Nacional de Rosario)

\section{Evaluaron este volumen}

Roberto Bárcena, María Teresa Boschin, Leonel Cabrera, Ulises Camino, María Rosa Carbonari, Carlos Ceruti, Horacio Chiavazza, Nicolás Ciarlo, Silvia Cornero, Eduardo Crivelli, Javier García Cano, Martín Gentinetta, María Laura Gili, Carlos Landa, Matilde Lanza, Melina Malandrino, Sebastián Pastor, Victoria Pedrotta, Josefina Piana, Mercedes Podestá, Mariano Ramos, Daniel Schavelzon, Diana Tamburini, Mónica Therrien, Mónica Valentini y María Teresa Boschin

\section{Diseño y diagramación}

Eugenia Reboiro

(eugenia.reboiro@gmail.com)

\section{Curadoría}

Ana Rocchietti e Irene Dosztal

Foto de tapa: Material arqueológico, del texto de Tapia et al.

\section{Propietario responsable:}

Facultad de Humanidades y Artes, Universidad Nacional de Rosario Centro de Estudios de Arqueología Histórica

Entre Ríos 758. Rosario, provincia de Santa

Fe (2000). Argentina.

Telf.: +54 (0341) 4802670

E-mail: ceahunr@gmail.com

Decreto Ley 6422/57 de Publicaciones

Periódicas 


\section{Índice}

Editorial

El origen del patrimonio histórico y su conservación integral en la contemporaneidad.

Yanina Aguilar

Prospección de basurales históricos de la ciudad de Rosario.

Soccorso Volpe y Gustavo Fernetti

Desconstrucción de un paisaje urbano. El Montevideo del extramuros colonial, aportes de la arqueología a su identidad actual. Ana Gamas

La Calota de Ameghino: reconsiderando un viejo tema desde la arqueología histórica.

Daniel Schávelzon

Análisis zooarqueológico de los restos hallados en una estructura sanitaria vinculada a momentos tempranos del actual barrio de Belgrano .71 Mario Silveira, Horacio Padula, Ricardo Orsini y Eva Bernat

La medida del tiempo: el reloj solar jesuita de La Cruz, provincia de Corrientes.

Fernando Oliva y María Cecilia Panizza

Sitio La Quinta: arqueología rural de campos serranos.

Flavio Ribero

Si no hay tabaco que no se note. Prácticas fumatorias en el fortin La Perra (1883-1885), La Pampa.

Alicia Tapia, Virginia Pineau y Melisa Ayelén Auge

El uso del tabaco y las pipas de caolin (clay pipes) en la frontera sur y oeste de la provincia de Buenos Aires en el siglo XIX.. .127

María del Carmen Langiano y Julio Fabián Merlo 
Patrimonio natural, urbano y arquitectónico de la costa en Mar del Plata. Federico Negroni

Huellas urbanas.

Ezequiel Serrot

Paisaje y patrimonio. La industria taninera en el siglo XX.

Provincia de Santa Fe.

Cristina Pasquali, Paola Milicic y Lara Ferré

Los desafíos de las arqueologías históricas latinoamericanas.

Andrés Zarankin

Sellos entre escombrales. Las lozas en el registro urbano

del Área Fundacional de Mendoza (siglo XIX y principios del siglo XX)

Lorena Puebla y Horacio Chiavazza

Evolución edilicia de la Plaza de Mayo de la Ciudad

de Buenos Aires (1580-1853).

Nicolás Ferrino

Gestión patrimonial en el rescate arqueológico y futuro Museo de Sitio

en Moreno 550, Ciudad Autónoma de Buenos Aires, Argentina.

María Eva Bernat, Ricardo Orsini, Horacio Padula y Mario Silvera 


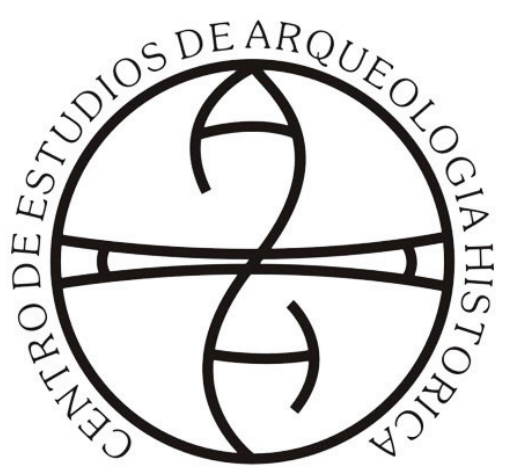

Centro de Estudios de Arqueología Histórica

Universidad Nacional de Rosario
Teoría y Práctica de la Arqueología Histórica

Latinoamericana | Año VIII. Vol. 9 | 2019

Revista del Centro de Estudios de Arqueología

Histórica, Facultad de Humanidades y Artes, Universidad Nacional de Rosario

https://teoriaypracticaah.unr.edu.ar/index.php/index ceahunr@gmail.com

ISSN en línea: 2591-2801

ISSN versión impresa: 2250-866X

\title{
LOS DESAFÍOS DE LAS ARQUEOLOGÍAS HISTÓRICAS
}

\section{LATINOAMERICANAS}

\author{
Andrés Zarankin*
}

Tradicionalmente las posiciones más ortodoxas en arqueología histórica en América han limitado este campo a presentar visiones que refuerzan y naturalizan pasados colonialistas. Originalmente, el foco de esta arqueología han sido los personajes y sitios considerados por las históricas oficiales como"importantes," dejando fuera las minorías y grupos subalternos. Estos trabajos en general tienen un carácter acrítico y suelen contener densas descripciones y clasificaciones de objetos y estructuras, que de manera esencial, son considerados patrimonio de una nación.

A partir de la década de 1990, en función de propuestas des-colonizantes como las de Pedro Funari, Tania Andrade Lima, Charles Orser, entre otros, la Arqueología Histórica comenzó a cambiar su foco de interés para ocuparse de la construcción de la Historia de los grupos sin historia dentro de los contextos locales de cada región (por eso el plural de "Arqueologías Históricas”).

La arqueología vista así es una forma de acción política (McGuire 2008) y como tal enfrenta las "metanarrativas" y discursos homogeneizantes, para ayudar a legitimar los grupos oprimidos, denunciar las injusticias y asumir un lugar activo en la lucha en pos un presente más democrático y pluralista (Funari 1997, Andrade Lima 1999, Gnecco 2012, Haber 2013, entre otros).

Si la construcción del "pasado" ha sido fuente de expansión del sistema de poder en el presente, su transformación en "otros" pasados, puede ser la base para el inicio de un cambio. Precisamente en esta presentación discuto a partir de la arqueología de la arquitectura (Stedman 1996, Zarankin 2002) una línea de estudio dentro de estas nuevas Arqueologías Históricas Latinoamericanas, en la que vengo trabajando en los últimos años, no solo como forma de dar visibilidad a las historias que nunca fueron contadas, sino también para entender las estrategias de reproducción del sistema.

Tomo como casos de análisis, lugares asociados a nuestra vida cotidiana, ya que como señala Foucault (1980) afirma que el proceso de reproducción del poder (o micro-poderes) funciona dentro de la esfera de

* Departamento de Antropología y Arqueología, UFMG, Brasil. zarankin@yahoo.com 
Teoría y Práctica de la Arqueología Histórica Latinoamericana | Año VIII. Vol. 9 | 2019

ISSN en línea: 2591-2801 | ISSN versión impresa: 2250-866X

lo que podemos denominar "situaciones cotidianas", y por lo tanto imperceptibles para las personas. Una de estas estrategias está asociada al control de la distribución de personas y cosas en el espacio (Foucault 1976). Así la arqueología, a partir de su capacidad para entender el universo material, puede contribuir a develar estas estrategias invisibles del sistema. Considero que este tipo de arqueología del pasado cercano (Buchli y Lucas 2002, Ruibal 2014)es una forma de desarrollar una arqueología "descolonizante" que permite generar cambios en el presente y así como una sociedad másdemocrática y justa.

\section{Arqueología Histórica y Sociedad Moderna}

La Arqueología Histórica Americana ha sido definida como el estudio de la conformación del Mundo Moderno (Orser y Fagan 1995) que comienza con la expansión europea (Falk 1991, Deetz 1991) y coincide con la consolidación del sistema capitalista y de un nuevo orden social (Johnson 1996). Así la conformación de la sociedad moderna ha sido postulada como un proceso de cambio que involucró diferentes aspectos de la vida cotidiana (Glassie 1975, Deetz 1977, Leone y Potter 1988, Beaudry et al. 1991, Johnson 1996, entre otros).

Las ideas de este nuevo orden social surgieron a partir de diversos estudios en las colonias inglesas en Norteamérica en el siglo XVIII (Leone et. al 1987, 1988, Little y Shackel 1989, Yentsch 1994, entre otros).Según estos la sociedad moderna se relaciona con el desarrollo de las relaciones capitalistas. Individualismo, segmentación, estandarización y consumismo son considerados conceptos clave para entender las bases de este nuevo sistema. (Orser 1996). Así, desde perspectivas arqueológicas se identificaron conjuntos de reglas que pueden ser aplicadas a la arquitectura, la cultura material y las formas de vida (Leone 1977, 1988, Beaudry 1989, McGuire 1991, Little y Shackel 1991, Yentsch 1991, Johnson 1996, entre otros).

Este modelo teórico es habitualmente empleado para explicar la conformación de "la sociedad moderna" aceptando su aplicabilidad en diferentes contextos geográficos (Orser 1996). Sin embargo asumir que los significados de las prácticas sociales y sus cambios difieren de contexto en contexto (Barrett 1988), implica la necesidad de explicar los cambios enfatizando la particularidad de distintos contextos locales (Zarankin y Senatore 2002). En este sentido Funari (et al. 1999: 15) señalan "it is necessary to maintain a critical perspective on the nature of inter-relationships between different societies and on any apparent tendencies towards cultural homogenization". Si nos limitamos a emplear, de forma acrítica, ideas y conceptos pensados para sociedades industriales para definir lo que es "sociedad moderna" y lo que no, Latinoamérica, salvo algunos casos, siempre seráperiférica y atrasada. Es necesario pensar la modernidad desde una escala local, no solo desde perspectivas globales sino también desde lógicas regionales. La modernidad es heterogéneaasí como las estrategias de reproducción de los sistemas de poder.

Como arqueólogos latinoamericanos tenemos la responsabilidad de desarrollar una arqueologíapolítica, crítica y anti-colonial. No una arqueología cerrada, sino abierta, que utilice todas las herramientas teóricas y metodologías que existen para pensar las características particulares de la conformación de nuestras sociedades modernas latinoamericanas.

\section{Arqueología de lo cotidiano en el mundo moderno}

Los discursos dominantes de la modernidad estuvieron fundamentados en un ideal de orden. Por este motivo, intentaron explicar la realidad mediante una racionalidad única. El pensamiento científico aportó las herramientas necesarias para alcanzar este objetivo. En este escenario, la historia se transformó en el 
resultado de un proceso de objetivación aparentemente neutral. A partir del mismo, una serie de situaciones excepcionales (generalmente, protagonizadas por sectores acomodados) se consideraron representativas de una época o período determinado. Este recorte invisibilizó la multiplicidad de expresiones que caracterizaron la cotidianeidad en el pasado. De la misma manera, naturalizó una serie de principios estructurantes que legitimaron diversas formas de poder en el presente.

La cotidianeidad comprende la mayor parte de la vida de las personas -independientemente de su condición social. Desde esta perspectiva, se presenta como el espacio y tiempo en que simultáneamente se construye, reproduce y transforma la sociedad. En resumidas cuentas, el mundo cotidiano modela las experiencias y prácticas, así como los significados que las personas otorgan a su accionar. Desde hace algunos años, los arqueólogos latinoamericanos han comenzado a discutir los rasgos que definieron la cotidianeidad de la sociedad moderna en contextos particulares (Andrade Lima 1993, 1997, 1999, Funari 1995, 1999, Orser y Funari 2001, Senatore 2001, Symanski 1998, 2001, Zarankin 2000, 2001, Zarankin y Senatore 2002, Salerno 2006). Algunas preguntas que intentaron abordar fueron: ¿Qué rasgos caracterizaron la cotidianeidad de la sociedad moderna en distintos momentos y lugares de América Latina? ¿Existen formas específicas en que los latinoamericanos podemos contar la historia cotidiana de nuestras propias sociedades? ¿De qué manera podemos contribuir los arqueólogos latinoamericanos a las discusiones sobre modernidad en un escenario académico global? ¿Cómo impactan los discursos sobre la vida en el pasado en la constitución de nuestras propias sociedades? ¿Qué tipo de personas se intentan construir a partir de estos discursos? ¿Qué mecanismos de poder se logran reproducir a partir de estas circunstancias? A través del análisis de la cultura material, estos investigadores han intentado conocer la diversidad de formas en que las personas se alimentaron, vistieron y habitaron el espacio en el pasado. De esta manera, la disciplina ha comenzado a enfrentar los discursos que -influenciados por el pensamiento moderno- produjeron un entendimiento generalizante y monolítico de la propia modernidad en América Latina.

\section{Arqueología de la Arquitectura}

Una de las características de la modernidad consiste en la fragmentación de espacios en un proceso que parece no tener fin. Surgen así nuevos límites que se materializan a través de la arquitectura, regulando nuestros movimientos y por lo tanto nuestras vidas. Con el tiempo esta organización del espacio pasa a formar parte del habitus (Bourdieu 1988) de las personas, hasta el punto de transformarnos en dependientes y reproductores de límites y de las ideologías asociadas a los mismos (Zarankin 2002).

Este tema siempre me intereso por lo que desde hace varios años vengo trabajando en una arqueología de la arquitectura ${ }^{1}$ (Stedman 1996) centrada en la construcción de los espacios que forman parte de nuestro cotidiano. Así desarrolle estudios en lugares como casas, escuelas, muros, instituciones bancarias, prisiones, entre otros(Zarankin 1999, 2000, 2002, 2005 a y b, 2008, 2012, Zarankin y Niro 2006).

En mi abordaje de las estructuras arquitectónicas, las personas son especialmente consideradas en su dimensión corporal (que es simultáneamente material). Ello se asocia a que el cuerpo es la única forma en que podemos "ser-en-el-mundo" (sensu Heidegger 1927). El cuerpo habilita las experiencias, y nos permite alcanzar un cierto conocimiento práctico del mundo ("un saber que está en las manos" -sensu Merleau-Ponty 1993 [1945]) a través de nuestro complejo sensorio-motor. Por su parte la arquitectura es considerada como una entidad agente (no un objeto pasivo, siempre sometido a nuestra contemplación). Por este motivo, su propia existencia puede solicitar nuestra respuesta; por ejemplo, que lo percibamos y recorramos de una manera determinada. Llegado este punto, vale la pena señalar que estas formas 
Teoría y Práctica de la Arqueología Histórica Latinoamericana | Año VIII. Vol. 9 | 2019

ISSN en línea: 2591-2801 | ISSN versión impresa: 2250-866X

de abordarla se encuentran guiadas por nuestras propias pautas culturales y posicionamientos sociales (Csordas 1990). Así, nunca pueden ser consideradas únicas u objetivas. Teniendo en cuenta lo expresado, resulta posible señalar que -cuando recorremos una estructura arquitectónica- se activan diversos mecanismos corporales que poseen un impacto en la constitución y construcción del habitus de las personas (Bourdieu 1988). Es precisamente a través del reconocimiento y la familiarización con estos "lugares" que al "in-corporar" el espacio en que habitamos, estamos también asimilando discursos no-verbales que muchas veces contribuyen a nuestra domesticación por el sistema (King 1980, Fletcher 1989, Monks 1992, Markus 1993, Kent 1990).

\section{Casas y Escuelas}

La arquitectura puede ser utilizada como un medio eficaz para controlar y "disciplinar" a las personas. De ésta manera, partiendo de entender a la arquitectura como una tecnología del poder (Foucault 1976), su control y manipulación puede ser vista como una estrategia del poder para perpetuarse. Tanto la morfología de las estructuras como la organización del espacio son elementos centrales para garantizar el funcionamiento del poder. A través de su control y manipulación el sistema construye categorías y estructuras mentales, de carácter "esencial", que tornan naturales y por lo tanto incuestionables sus bases ideológicas.

En este contexto, cobran especial importancia aquellas estructuras arquitectónicas relacionadas con los procesos de socialización de las personas (Samson 1990, Parker Pearson y Richards 1994). Entre estas, Bourdieu (1977) destaca la vivienda familiar y la escuela. Estas revisten especial importancia ya que es allí donde las personas incorporan la mayoría de los esquemas mentales que los acompañará por el resto de su vida (habitus primario).

A pesar de que existen diversos estudios sobre casas y escuelas en las ciencias sociales y humanas, estos se han centrado en cuestiones como la conformación de las estructuras parentales, relaciones familiares, curriculum escolar y organización de la escuela, entre otros. Prácticamente no existieron estudios sobre la materialidad de estos lugares. Así, a fines de la década de 1990 realice pesquisas sobre los procesos de transformación arquitectónico-espacial en casas familiares y escuelaspúblicas primarias de la Ciudad de Buenos Aires (Zarankin1999, 2000).

Para realizar este análisistrabaje principalmente con el modelo Gamma de Hillier y Hanson (1984) conjuntamente con los índices planteados por Richard Blanton (1994). Este método permitió generar una cuantificación y una comparación entre estructuras arquitectónicas (tomando variables como cantidad de habitaciones, conexiones entre habitaciones, circuitos de circulación, aislamiento de las habitaciones, distancia la exterior, entre otras) -Fig.1.

A través del tiempo pude observar un patrón común en el desarrollo de casas y escuelas de la ciudad de Buenos Aires, asociado a cambios en el sistema capitalista. En el caso de las escuelas públicas primarias, concebidas a mediados del siglo XIX desde un modelo panóptico, su transformación fue simplemente ir aumentando su tamaño formando cadenas de panópticos conectados entre sí por corredores, pasillos o escaleras (Figura 1). Los cambios estilísticos en la fachada, en la decoración o en el estilo de los edificios fueron más que nada agiornamientos para generar una sensación de que la escuela se modernizaba con el tiempo. 


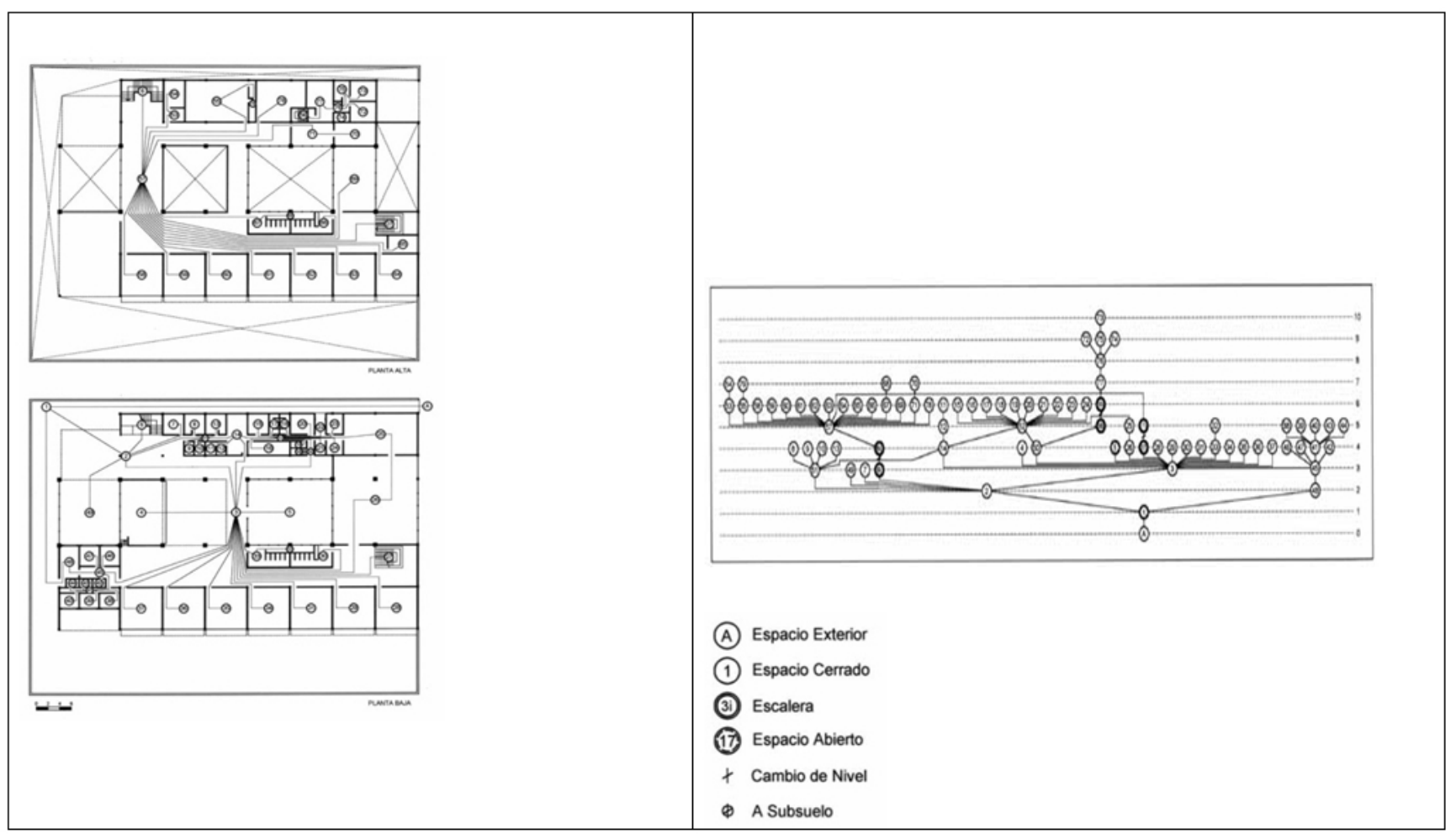

Figura 1- Planta de una escuela de la década de 1960 en la que puede observarse una estructura basada en un encadenamiento de panópticos.

Por su vez en el caso de las casas familiares, enmascarado debajo de un proceso "evolutivo" hacia modelos más confortables y funcionales, lo que existe es un aumento en el control sobre las personas. Esto resulta claro en la vivienda moderna, donde los espacios y sus funciones están predeterminados, por ejemplo: comedor, escritorio, garaje, habitación, living, etc. (Figura 2). Sus características espacio-morfológicas dificultan la posibilidad de poder otorgar funcionalidad a un espacio según las propias necesidades. De esta manera los ocupantes se ven restringidos y condicionados en los momentos de decisión. Al mismo tiempo, la casa distribuye sus ocupantes en función de jerarquías de poder. Nadie duda que la suite sea para los padres, y el segundo mejor cuarto para el hermano mayor o la hija preferida del padre. Este mapa de poder se va modificando, siempre sobre los mismos criterios, a medida sus habitantes van abandonando la casa. 


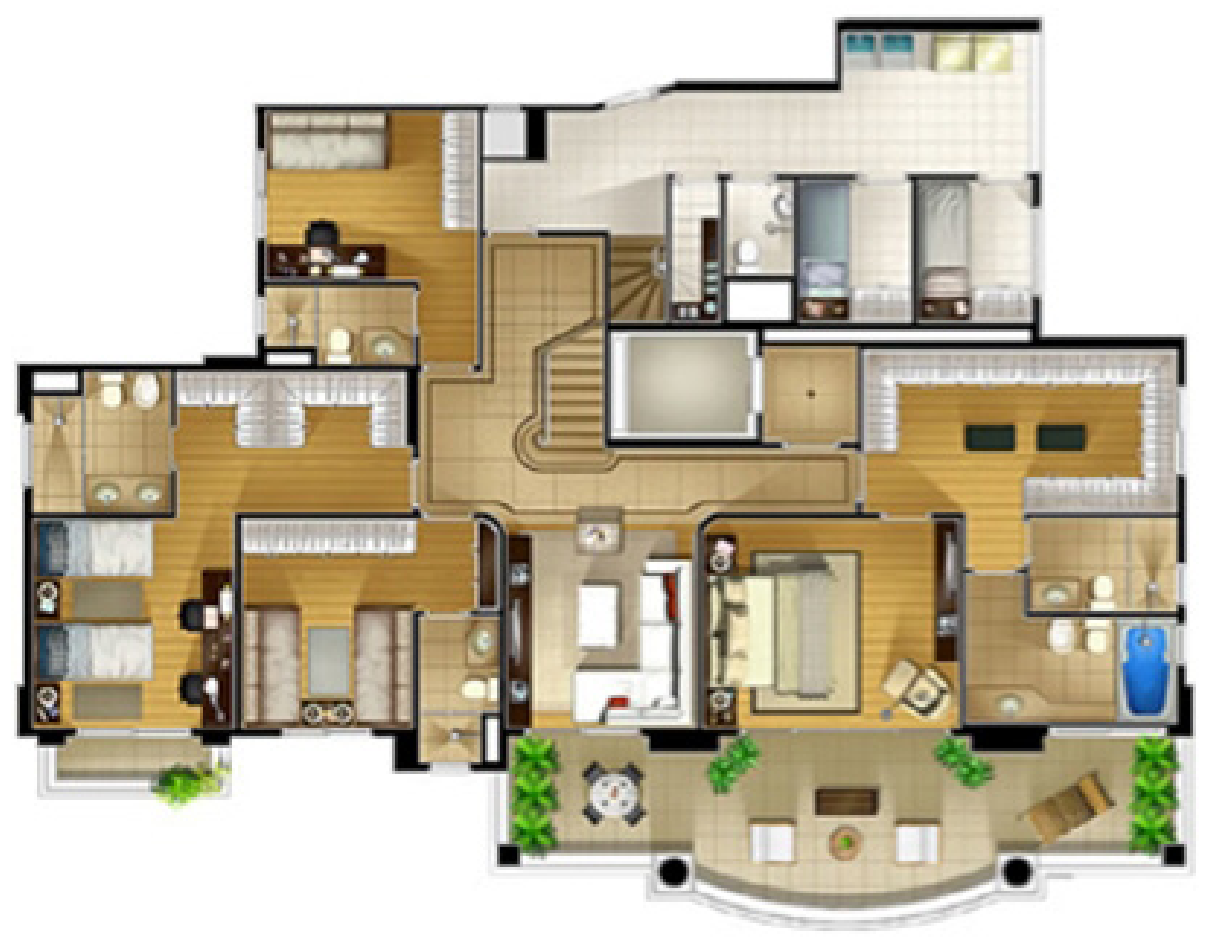

Figura 2- Planta de una casa "moderna”, en la que se pueden observar principios de organización jerárquicos, restrictivos y autoritarios.

Podemos pensar entonces que casas y escuelas son dispositivos del poder que funcionan como discursos no-verbales que disciplinan y adoctrinan sus usuarios para ser funcionales a las necesidades del sistema. Como muestra el cuadro de la figura 3, existe una relación directa entre sistema (ya sea a partir de una visión marxista-economicista o asociada a sistemas de poder, y las transformaciones en escuelas y casas). 
Teoría y Práctica de la Arqueología Histórica Latinoamericana | Año VIII. Vol. 9 | 2019

ISSN en línea: 2591-2801 | ISSN versión impresa: 2250-866X

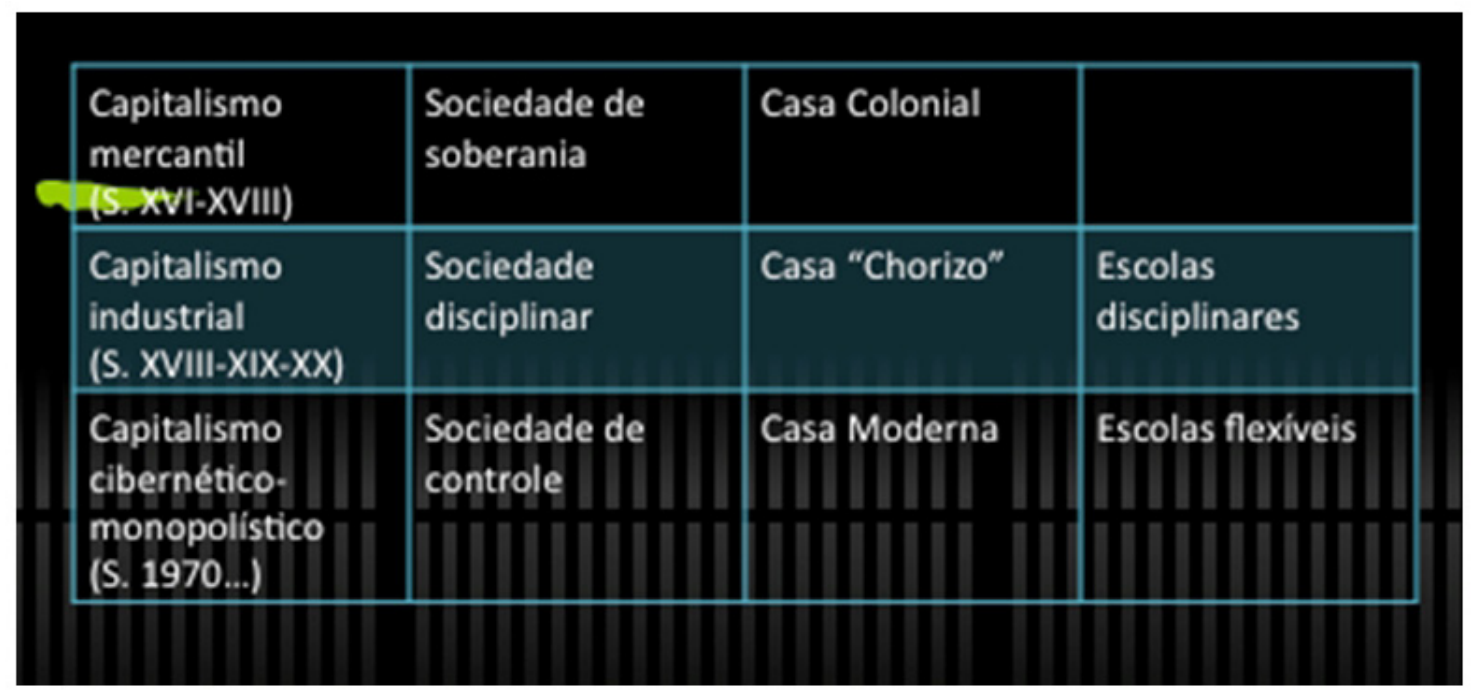

Figura 3- Cuadro comparando casas, escuelas y momento histórico

\section{Centros Clandestinos de Detención (CCD)}

Considero que la arqueología histórica latinoamericana se encuentra cada vez más comprometida con las causas sociales (Zarankin, Salerno y Perosinio 2012). Desde esta posición, se halla dispuesta a asumir la responsabilidades, como por ejemplo la de investigar los sucesos ocurridos durante las dictaduras militares que asolaron la región en la segunda mitad del siglo XX (Funari y Zarankin 2002). A pesar de los intentos de ocultamiento y distorsión de quienes participaron en los gobiernos autoritarios, los resultados que se estánobteniendo permiten ser optimistas y confiar en que otra historia puede ser contada.

Si bien los trabajos denominados como arqueología de la represión (Funari y Zarankin 2002) continúan siendo minoritarios, el panorama ha comenzado a cambiar. En líneas generales, la arqueología no sólo viene contribuyendo a comprender el funcionamiento de los sistemas desaparecedores de personas. También ha colaborado con la construcción de una memoria material del genocidio. El desarrollo de nuevos espacios de producción en América Latina ha permitido generar un diálogo fructífero entre los profesionales, demostrando las semejanzas y diferencias de los procesos represivos en distintos países de la región (Zarankin y Salerno 2011). Asimismo, estos espacios de trabajo han abierto la posibilidad de sistematizar un conjunto de estudios que anteriormente se presentaba como fragmentario y desarticulado. Actualmente, el desafió es asegurar la continuidad de los trabajos y fomentar la creación de nuevas investigaciones.

Los diversos estudios en Arqueología de la represión desde sus orígenes en la década de 1980, comparten un compromiso político-social con la búsqueda de la verdad y, de manera general, pueden ser agrupados en 5 líneas principales (Zarankin, Salerno y Perosinio 2012): 1) las reflexiones teóricas sobre arqueología de la represión, memoria y usos del pasado; 2) la recuperación e identificación de restos de personas desaparecidas; 3) el estudio de centros clandestinos de detención; 4) el análisis de objetos asociados a la represión; 5) el abordaje de casos emblemáticos.

En mi caso específico trabaje en 2002 en la excavación de un campo de concentración de la dictadura argentina, conocido como "Club Atlético" (sobrenombre derivado de las iniciales del verdadero nombre del lugar - "Centro Antisubversivo"). Este funcionó por algunos meses de 1977 en el interior de un de- 
pósito de la Policía Federal Argentina. El edificio se localizaba en la actual intersección de la Av. Paseo Colón y la Autopista 25 de Mayo, a tan sólo unos pocos minutos del microcentro porteño. Por allí pasaron alrededor de 1500 personas en calidad de detenidos, la mayor parte de los cuales continua desaparecida. El Club Atlético tenía capacidad para albergar hasta 200 individuos al mismo tiempo, y poseía espacios destinados a la tortura. Hacia fines de 1977, el edificio donde funcionaba el ex centro de detención fue demolido, y en su lugar se procedió a construir un tramo de una autopista. Las ruinas del Club Atlético permanecieron inaccesibles por 25 años. Frente a estas circunstancias, los sobrevivientes y organismos de derechos humanos reclamaron la realización de excavaciones en el lugar desde el retorno de la democracia (Figura 4).

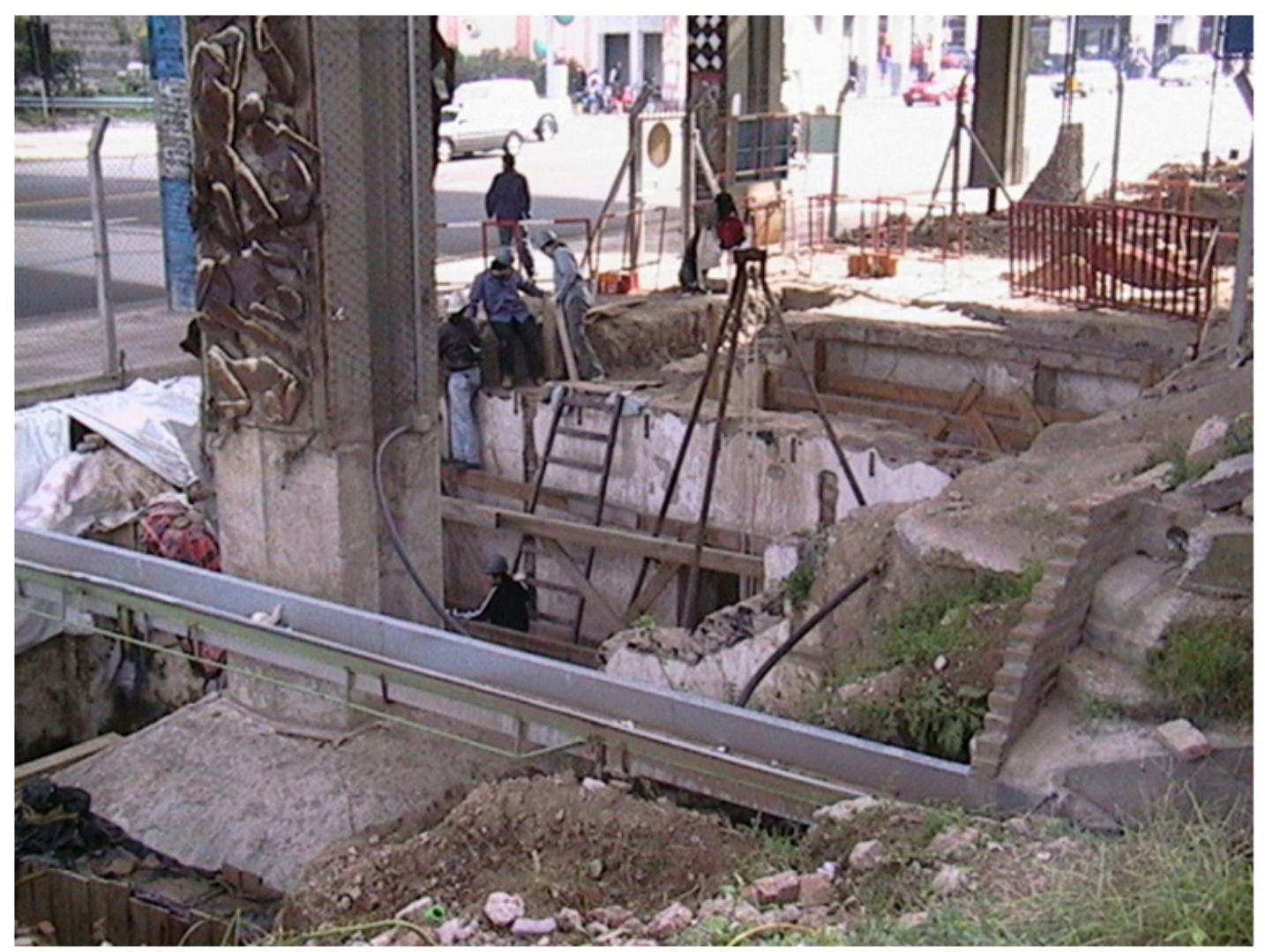

Figura 4- Vista de los trabajos de excavación en el “Club Atlético” (2003)

En 2003 (y después de una primera experiencia que resultó interrumpida en 2002), la Secretaría de Derechos Humanos del Gobierno de la Ciudad convocó a un nuevo concurso de proyectos para la intervención arqueológica del lugar. En ese escenario se seleccionó la propuesta "Arqueología como memoria: Intervenciones arqueológicas en el centro clandestino de detención y tortura Club Atlético" (Bianchi Villelli y Zarankin 2002).

Los objetivos generales del proyecto fueron estudiar la organización espacial del antiguo centro, y colaborar con la construcción de una memoria material de lo sucedido. La ejecución de las tareas dependió de un comité de manejo, en el que participaron sobrevivientes del centro, familiares de las víctimas, representantes de organizaciones de derechos humanos, ingenieros, antropólogos, arqueólogos, funcionarios del 
gobierno. La experiencia fue estimulante, en tanto los arqueólogos -frecuentemente habituados a dirigir proyectos caracterizados por los estándares de una ciencia neutral - se transformaron en parte de un esfuerzo colectivo por dilucidar uno de los aspectos más oscuros de la represión en el país (Zarankin y Niro 2006).

Dentro de la línea de trabajo teórico-metodológica que venía desarrollando en arqueología de la arquitectura, utilizando una planta producida por los propios detenidos, aplique los modelo Gamma de Hillier y Hanson (1984) y los índices de Blanton (1994). La utilización de los mismos permitió observar como elemento organizativo del espacio un parámetro de maximización y operatividad de los procedimientos represivos. Funcionan como base de esta estructura, una circulación restringida y controlada, además de un profundo aislamiento de los ambientes.

Por otra parte el espacio del "CA" puede ser dividido en 2 ejes (Figura 5). Un sector superior (que ocupa aproximadamente un $20 \%$ de la superficie total), que podemos denominar como el centro burocrático del CCD. Existe otro posterior, que aloja a los prisioneros y donde se ubican las salas de tortura. Esta organización divide y clasifica a las personas dentro del mismo, delimitando espacios de circulación y permanencia de los detenidos.

Figura 5- Esquema de la organización del espacio en el Club Atlético

\begin{tabular}{|l|}
\hline Burocracia \\
\hline $\begin{array}{l}\text { Salas de detención } \\
\text { colectivas }\end{array}$ \\
\hline Celdas \\
\hline Salas de Tortura \\
\hline Celdas \\
\hline
\end{tabular}

Así la instalación de las salas de tortura en un espacio central entre los calabozos permite por un lado minimizar el traslado de los detenidos en el espacio, al mismo tiempo que sus gritos puedan ser oídos por quienes en ese momento están en las celdas.

Existe también un elemento simbólico asociado a la organización del espacio. Así a medida que se avanza hacia el interior del CCD el nivel de suplicio va aumentando. Imaginemos que el prisionero no puede ver ya que solían tener vendas o capuchas en sus cabezas, pero si experimentar ese espacio a través de los sentidos. Los olores de cuerpos y fluidos humanos, la humedad y la falta de ventilación del subsuelo, el calor y el frío, los gritos y llantos de los otros detenidos, la dureza de las paredes y el piso -donde eran colocados-. Se trata de una estructura para ser percibida de maneras alternativas a la visión, a través de sentidos como el tacto, el sonido o el olor. Su centro, su corazón, es la sala de tortura, la que de alguna manera representa la materialización del sadismo con la que fue proyectado el CCD (Figura 6). 


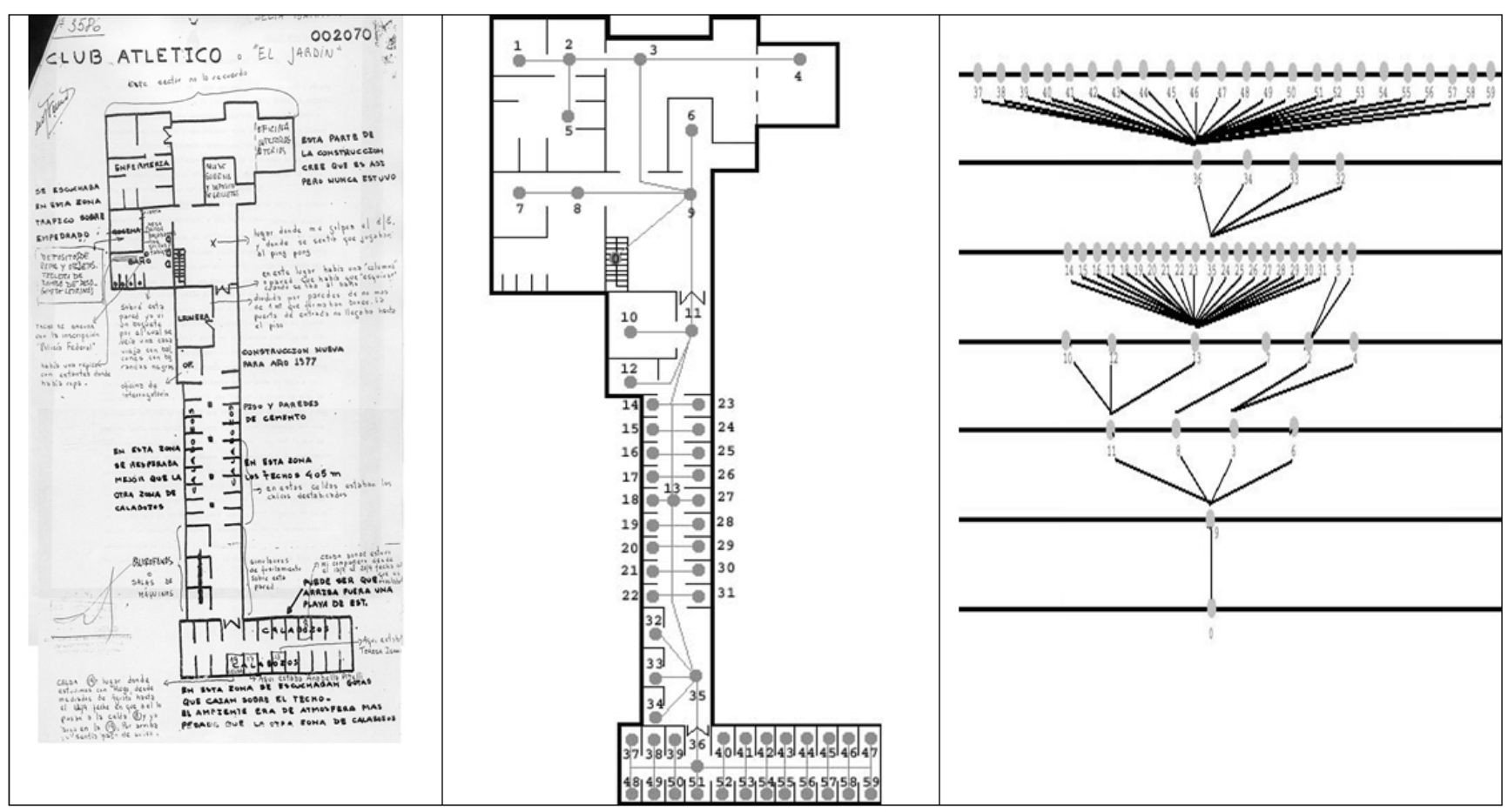

Figura 6- Planta generada por los propios sobrevivientes en función de sus recuerdos (en Benítez, Enríquez y Di Ciano 2001:10) y Aplicación del modelo Gamma

En síntesis el estudio mostró que los centros clandestinos de detención son al mismo tiempo, dispositivos del poder destinadosa destruir cuerpos y mentes, y metáforas materiales que codifican discursos autoritarios (Zarankin y Niro 2006). Su análisis revela el plan sistemático de aniquilación de toda disidencia generada desde el gobierno militar.

\section{Palabras finales}

A partir de la arqueología de la arquitectura una de mis preocupaciones principales ha sido contribuir a profundizar la crítica arqueológica sobre la cotidianeidad del presente como instrumento de reproducción del poder, así como sus múltiples conexiones en el ámbito latinoamericano. Al mismo tiempo me propongo traer posibilidades para cuestionar el corral epistemológico generado a partir de las dicotomías modernas, traer y discutir las relaciones periféricas, o incluso, como plantea Alejandro Haber, cuestionar los cuestionamientos.

Considero que las arqueologías históricas latinoamericanas están trabajando para delinear un perfil teórico propio, acordes a sus propias tradiciones y contextos socio-políticos.Son las nuevas generaciones de arqueólogos las que están contribuyendo a fortalecer estas identidades locales y regionales, transformando la disciplina en un instrumento útil para la crítica y la transformación social.

\section{Agradecimientos}

A Melisa Salerno por su lectura crítica y comentarios. A las organizadoras del VIII Congreso Nacio- 
nal de Arqueología Histórica Argentina, Dra. Ana María Rocchietti e Dra. Irene Dosztal. Finalmente al Departamento de Antropología y Arqueología de la UFMG

\section{Notas}

"Arqueología de la arquitectura", es una línea reciente de investigación que comprende todas aquellas investigaciones enfocadas al estudio de la arquitectura desde una perspectiva arqueológica (Steadman 1996). Este tipo de abordajes, ofrece una nueva perspectiva de análisis para abordar aspectos relacionados con la conformación del entorno humano.

\section{Bibliografía}

Andrade Lima, T. (1993) Arqueologia histórica no Brasil: balanço bibliográfico (1960-1991). Anais do Museu Paulista, Nova Série, História e Cultura Material 1: 225-262.

Andrade Lima, T. (1997) Chá e simpatía: Uma estratégia de gênero no Rio de Janeiro oitocentista. Anais do Museu Paulista: História e Cultura Material 5: 93-129.

Andrade Lima, T. (19999 El huevo de la serpiente: Una arqueología del capitalismo embrionario en el Rio de Janeiro del siglo XIX. En Acuto, F. y Zarankin, A. (eds.): Sed Non Setiata: teoría social en la arqueología latinoamericana contemporánea. (pp. 189-238) Buenos Aires: Ediciones Del Tridente.

Barrett, J., (1998) Fields of discourse. Reconstituting a social archaeology, Critique of Anthropology 7(3), pp. 5-16.

Beaudry, M. C. (1989) The Lowell boottmills complex and itshousing: Material expressions of corporateideology. Historical Archaeology 23 (1): 19-32.

Beaudry, M., Cook, L. y Mrozowski, S. (1991) Artifacts and active voices. Material culture as social discourse, en R. McGuire y R. Paynter (eds.) The Archaeology of Inequality, (pp. 150-191) London: Blackwell.

Benítez, M., Enríquez, Z. y Di Ciano, L. (2001) El Club Atlético. Revista de la Asociación de ex detenidos desaparecidos 1 (2): 1-18.

Bianchi Villelli, M. y Zarankin A. (2002) Arqueología como memoria. Intervenciones arqueológicas Intervenciones arqueológicas en el Centro Clandestino de Detención y Tortura "Club Atlético". Plan de Trabajo aceptado por la Comisión de Trabajo y Consenso del Proyecto "Recuperación de la memoria del Centro Clandestino de Detención y Tortura Club Atlético". Dirección General de Derechos Humanos. Gobierno de la Ciudad Autónoma de Buenos Aires. Ms.

Blanton, R. (1994) Housesend Households. New York: Plenum Press.

Bourdieu, P. (1977) Outline of a Theory of Practice. Cambridge: Cambridge University Press.

Buchli, V. y Lucas, G. (2001) Archaeologies of the Contemporary Past.Routledge,

Csordas, T. (1990) Embodiment as a paradigm for anthropology. Ethos 18 (1): 5-47. 
Bourdieu, P. (1988) La Distinción. Madrid, Taurus.

Deetz, J. (1977) In Small Things Forgotten. New York: Anchor Books.

Deetz, J. (1991) Archaeological evidence of sixteenth and seventeenth century encounters. En Falk, L. (ed.): Historical Archaeology in Global Perspective. (pp. 1-10) Washingto:, Smithsonian Institution.

Falk, L. (ed.) (1991). Historical Archaeology in Global Perspective. Washington: Smithsonian Institution.

Fletcher, R. (1989) “The Messages of Material Behaviour: a Preliminary Discussion of Non-verbal Meaning”. En Hodder, I. (ed.): The Meaning of the Things: Material Culture and Symbolic Expression. (pp. 33-39) Londres: Harper Collings.

Foucault, M. (1976) Vigilar y Castigar. El Nacimiento de la Prisión. México, Siglo XXI.

Foucault, M. (1980) Power/Knowledge: Selected Interviews and Other Writings, 1972-1977. New York: Phanteon Book

Funari. P. (1995) A cultura material e a construção da mitología Bandeirante: Problemas da identidade nacional brasileira. Ideais 1: 29-48.

Funari. P. (1997) Archaeology, History and Historical Archaeology in South America. International Journal of Historical Archaeology 1: 137-148.

Funari. P. (1999) Historical Archaeologyfrom a WorldPerspective. En Funari, P., Hall, M. y Jones, S. (ed.): Historical Archaeology: Back fromthe Edge. (pp. 37-66) Londres: Routledge.

Funari, P. y Zarankin, A. (2002) A social archaeology of housingfrom a Latin American perspective: a case study. Journal of Social Archaeology 3 (1):23-45.

Glassie, H. (1975) Folk Housing in Middle Virginia. Knoxville: University of Tennessee Press.

Gnecco, C. (2012) Arqueología Multicultural. Notas intempestivas. Complutum 23 (2): 93-102.

Haber, A. (2013) Anatomía Disciplinaria y Arqueología Indisciplinaria. Revista Arqueología 19. Pp. 53-60.

Heidegger, M. (1927) El Ser y el Tiempo. Buenos Aires: Planeta.

Hillier, B. y Hanson J. (1984) The Social Logic of Space. Cambridge, Cambridge University Press.

Johnson, M. (1996) Anarchaeology of capitalism. Oxford, Blackwell.

Kent, S. (1990) Domestic Architecture and the Use of Space. New Direction in Archaeology. Cambridge: Cambridge UniversityPress.

King, A. (ed.) (1980) Buildings and Society; Essays on the Social Development of the Built Environment. Boston: Routledge-Keagan Paul.

Leone, M. (1977) The New Mormon Temple in Washington DC. En Ferguson, L. (ed.): Historical Ar- 
Teoría y Práctica de la Arqueología Histórica Latinoamericana | Año VIII. Vol. 9 | 2019

ISSN en línea: 2591-2801 | ISSN versión impresa: 2250-866X

chaeology and the Importance of Material Things. Arizona, Society for Historical Archaeology. Special Publication Series (2):43-61.

Leone, M., Potter, P. y Shackel, P. (1987) Towards a criticalarchaeology. Current Anthropology 28 (3): 823-832.

Leone, M. y Potter P. (eds.) (1988) The Recovery of Meaning: Historical Archaeology in the Eastern United States. Washington D.C., Smithsonian Institution Press.

Little, B. y Schackel, P. (1989) Scales of Historical Anthropology: An Archaeology of Colonial Anglo America. Antiquity 63: 495-509.

Little, B. y Schackel, P. (1991) Post-Processual Approaches to meaning and uses of material culture in Historical Archaeology. Historical Archaeology 26: 5-11.

Markus, T. (1993) Buildings and Power; Freedom and Control in the Origin of Modern Buildings Types. Oxford: Blackwell.

McGuire, R. H. (1991) Building Power in Cultural Landscape of Broome County, New York 1880 to 1940”. En McGuire, R. H. y Paynter, R. (eds.): The Archaeology of Inequality. (pp.102-124) Massachusetts: Blackwell.

McGuire, R. H. (2008) Archaeology as PoliticalAction. Londres, University of California Press.

Merleau-Ponty, M.1993 [1945]. Fenomenología de la percepción. México/Buenos Aires: Fondo de Cultura Económica

Monks, G. (1992) Architectual Simbolism and Non-verbal communication at Upp.er Fort Garry. Historical Archaeology 26 (2): 37-57.

Orser Jr, Ch. (1996) A Historical Archaeology of the Modern World. New York, Plenum Press.

Orser Jr, Ch. y Fagan, B. (1995) HistoricalArchaeology. New York, Harper Collins.

OrserJr, Ch. y Funari, P. (2001) Archaeology and Slave Resistance and Rebellion. World Archaeology 33: 61-72.

Parker Pearson, M. y Richard, C. (eds.) (1994) Architecture and Order. Approaches to Social Space. Londres: Routledge.

Ruibal, A. G. (2014) Returning to Where We Have Never Been: Excavating the Ruins of Modernity. En Olsen, B. y Pétursdóttir, P. (eds.): Ruin Memories Materialities, Aesthetics and the Archaeology of the Recent Past. (pp. 367-389) London: Routledge.

Samson, R. (comp.) (1990) The Social Archaeology of Houses. Edinburgh: University Press.

Salerno, M. (2006) Arqueología de la Indumentaria. Prácticas e Identidad en los Confins del Mundo Moderno (Antártida, Siglo XIX). Buenos Aires: Del Tridente.

Stedman, S. (1996) Recent Research in the Archaeology of Architecture: Beyond the Foundations. Journal of Archaeologica IResearch 4 (1): 51-93. 
Teoría y Práctica de la Arqueología Histórica Latinoamericana | Año VIII. Vol. 9 | 2019

ISSN en línea: 2591-2801 | ISSN versión impresa: 2250-866X

Symanski, L. C. P. (1998) Espaço privado e vida material em Porto Alegre no século XIX. Porto Alegre: EDIPUCRS.

Symanski, L. C. P. (2001) Exposição e Isolamento: Práticas de descarte de refugo e mudanças de visão de mundo em um ambiente rural - o sítio Fazenda Camurugi (BA). Revista de Divulgação Científica 4: $113-137$.

Yentsch, A. (1991) Chesapeake artefacts and their culture context: pottery and the food domain. Post-Medieval Archaeology 25: 25-72.

Yentsch, A. (1994) A Chesapeake family and their slaves. Anstudy in Historical Archaeology. Cambridge: Cambridge University Press.

Zarankin, A. (1999) Casa Tomada; sistema, poder y vivienda domestica. En Zarankin, A. y Acuto, F. (eds.): Sed Non Satiata; Teoría Social en la Arqueología Latinoamericana Contemporánea. (pp. 239-272) Buenos Aires: Del Tridente.

Zarankin, A. (2000) Arqueología de la arquitectura. Anotherbrick in thewall. Revista do Museu de Arqueologia e Etnologia da Universidade de São Paulo. Pp. 119-128.

Zarankin, A. (2002) Paredes que Domesticam: Arqueologia da Arquitetura Escolar Capitalista; O caso de Buenos Aires. Campinas, Centro de Historia da Arte e Arqueologia (IFCH-UNICAMP).

Zarankin, A. (2005 $)$ Arqueología de la Arquitectura, modelando al individuo disciplinado en la sociedad capitalista. Revista de Arqueología Americana 22 (1): 25-41.

Zarankin, A. (2005b) "Walls of Domestication": Archaeology of thearchitecture of capitalistelementarypublicschools; The case of Buenos Aires. EnFunari, P.,Zarankin, A. y Stovel, E. (orgs.): Global Archaeological Theory: contextual voices and contemporarythoughts. (pp. 237-264) New York: Kluwer-Plenum.

Zarankin, A. (2008) Los Guardianes del Capital; Arqueologia de la Arquitectura de los Bancos de la Ciudad de Buenos Aires. En Acuto, F. y Zarankin, A. (orgs.): Sed Non Satiata II, acercamientos sociales en la arqueología latinoamericana. (pp. 325-339)Córdoba: Encuentro Grupo Editor.

Zarankin, A. (2012) Corpos congelados: umaleitura metafórica de paredes e muros em Belo Horizonte, MG. En Andrade, R., Macedo, J. y Terra, C. (org.): Arqueologia na paisagem: novos valores, dilemas e instrumentos. (pp. 18-33) Rio de Janeiro: Rio Books.

Zarankin, A. y Niro, C. (2006) La materialización del sadismo: Arqueología de la arquitectura de los centros clandestinos de detención de la dictadura militar argentina (1976-1983). En Funari, P. y Zarankin, A. (comp.): Arqueología de la Represión y Resistencia en América Latina (1960-1980). (pp. 159-182) Córdoba: Brújas.

Zarankin, A. y Senatore M. (orgs.) (2002) Arqueologia da Sociedade Moderna na América do Sul: Cultura Material, Discursos e Práticas. Buenos Aires: Ediciones del Tridente.

Zarankin, A. y M. Salerno (2011) The Engineering of Genocide: An Archaeology of Dictatorship in Argentina. En Myers, A. y Moshenska, G. (eds.): Archaeologies of Internment, One World Archaeo- 
Teoría y Práctica de la Arqueología Histórica Latinoamericana | Año VIII. Vol. 9 | 2019

ISSN en línea: 2591-2801 | ISSN versión impresa: 2250-866X

logy. (pp. 207-227) New York: Springer.

Zarankin, A., Salerno, M.y Perosino, M. (2012) Arqueologías de la clandestinidad. Una revisión de los trabajos efectuados en los centros de detención clandestinos de la última dictadura militar en Argentina. RUHM 2: 1-36. 